\title{
Globe
}

Revue internationale d'études québécoises

\section{Allan Greer, La Nouvelle-France et le Monde, traduit de l'anglais par Hélène Paré, Montréal, Les Éditions du Boréal, 2009}

\section{François-Joseph Ruggiu}

Volume 13, numéro 2, 2010

URI : https://id.erudit.org/iderudit/1001137ar

DOI : https://doi.org/10.7202/1001137ar

Aller au sommaire du numéro

Éditeur(s)

Globe, Revue internationale d'études québécoises

ISSN

1481-5869 (imprimé)

1923-8231 (numérique)

Découvrir la revue

Citer ce compte rendu

Ruggiu, F.-J. (2010). Compte rendu de [Allan Greer, La Nouvelle-France et le Monde, traduit de l'anglais par Hélène Paré, Montréal, Les Éditions du Boréal, 2009]. Globe, 13(2), 195-198. https://doi.org/10.7202/1001137ar d'utilisation que vous pouvez consulter en ligne.

https://apropos.erudit.org/fr/usagers/politique-dutilisation/ 


\title{
RECENSIONS
}

\author{
Frédéric Rondeau (éd.)
}

\section{Allan Greer \\ La Nouvelle-France et le Monde, traduit de l'anglais par Hélène Paré, Montréal, Les Éditions du Boréal, 2009.}

Depuis la publication en 1985 de sa thèse, Peasant, Lord, and Merchant: Rural Society in Three Quebec Parishes: 1740-1840, traduite en français en 2000, l'historien canadien anglophone Allan Greer, professeur à l'Université de Toronto puis à l'Université McGill, a construit un parcours de recherche original. L'ouvrage publié en 2009 par les Éditions du Boréal sous le titre La Nouvelle-France et le monde, permet au public francophone d'en mieux mesurer la diversité, mais aussi la cohérence, puisqu’il réunit une série de onze articles et communications, traduits de l'anglais, et rédigés de 1977 à 2006. La plupart d'entre eux datent, en fait, de la première moitié des années 2000 et ils sont précédés d'une introduction inédite.

Les premiers travaux d'Allan Greer, à la fin des années 1970 et au début des années 1980, ont été consacrés à l'histoire sociopolitique de la vallée du Richelieu durant la période qui va des dernières années du Régime français à la Révolte des Patriotes, et qui est, sans nul doute, une des plus complexes de l'histoire des régions qui ont formé le Canada. Les chapitres neuf et dix de La Nouvelle-France et le monde, renvoient directement à ce temps de la recherche d'Allan Greer. Le chapitre neuf, rédigé en 1977, explore, à partir d'un microévènement, la brève mutinerie en 1744 des soldats de la forteresse de Louisbourg, l'organisation de la société militaire de l'île Royale. Il est particulièrement intéressant de le lire aujourd'hui en parallèle avec la belle étude de Louise Dechêne, Le peuple, l'État et la guerre au Canada 
sous le Régime français (Boréal, 2008). Le chapitre dix, paru en 1980, est consacré à une analyse de la fonction politique des fabriques paroissiales dans la vallée du Richelieu à la fin du XVIII ${ }^{e}$ siècle et au début du XIX ${ }^{\mathrm{e}}$ siècle. Il complète certains développements de Peasant, Lord, and Merchant, en mettant en avant la persistance dans cette région du rôle des habitants, simples cultivateurs, dans le fonctionnement de ces institutions rurales, aux dépens des élites locales. Ces deux chapitres annoncent aussi l'intérêt d'Allan Greer pour la formation de l'État et ses liens avec le changement social que l'on retrouve dans Colonial Leviathan: State Formation in Mid-Nineteenth Century Canada, le collectif qu'il a publié en 1992 avec Ian Radforth ainsi que, naturellement, dans The Patriots and the People. The Rebellion of 1837 in Rural Lower Canada, paru en 1993.

Dans la seconde moitié des années 1990, les thématiques d'Allan Greer ont considérablement évolué. Il s'est de plus en plus intéressé à une histoire religieuse renouvelée à la fois par les apports de l'histoire culturelle (en particulier par l'intermédiaire des travaux sur les hagiographies médiévales) ou encore par l'étude de la formation des identités individuelles. Il a délaissé alors les voies traditionnelles de l'histoire sociale, d'ailleurs remises en cause à cette époque aussi bien en Europe qu'en Amérique du Nord, pour emprunter aux méthodes de l'anthropologie et de la théorie littéraire (voir, en particulier, p. 79-83). La Nouvelle-France, un des lieux emblématiques de la rencontre et de la confrontation entre Européens et Autochtones, lui a alors fourni un terrain d'exploration particulièrement fécond. Six chapitres, rédigés entre 2000 et 2006, éclairent le lecteur francophone, qui avait pu en prendre connaissance par le biais de la traduction, dès 2007, de Mohawk saint: Catherine Tekakwitha and the Jesuits, paru en anglais en 2005, sur ce véritable tournant intellectuel d'Allan Greer qui concerne aussi bien l'échelle de sa réflexion que ses thématiques.

Trois chapitres (7, 8 et 11) replacent l'action des Jésuites en Amérique du Nord à la fois dans son contexte culturel et dans son contexte hémisphérique, en particulier par la comparaison entre les missions jésuites au Paraguay et en Nouvelle-France. L'étude de la vie du père Chaumonot, retracée à partir de son autobiographie spirituelle, est particulièrement passionnante. Allan Greer insiste sur la capacité de ce Jésuite à investir les cultures dans lesquelles il a été amené à s'immerger, y compris la culture huronne, au point de transformer considérablement son identité personnelle et jusqu'à son nom. Trois autres chapitres $(4,5$ et 6$)$ mettent en perspective la vie de Catherine Tekakwitha, morte en 1680, et dont les Jésuites du Canada, en particulier le père Claude Chauchetière, ont promu le culte populaire. Le 
chapitre quatre revient sur la notion de conversion dans le contexte amérindien et replace le catholicisme dans l'histoire spirituelle des Iroquois; le chapitre cinq resitue la vie de Catherine Tekakwitha à Kahnawake, un village d'Iroquois chrétiens, établi près de Montréal, au sein de celles des autres "saints» de la Nouvelle- France; le chapitre six, enfin, analyse le rôle de la traduction en espagnol de la vie de la "sainte» iroquoise dans les controverses religieuses livrées au Mexique au début du XVIII siècle.

Au vu de l'ampleur des tournants conceptuels pris par Allan Greer, qui s'est, par ailleurs, aussi livré à l'exercice de la synthèse dans sa Brève histoire des peuples de la Nouvelle-France, traduite en 1998, il n'est pas étonnant qu'il ait développé une sensibilité particulière pour l'historiographie. Outre l'introduction, les premiers chapitres $(1,2,3)$ de ce volume sont donc consacrés à la mise en perspective des évolutions récentes de l'historiographie de la Nouvelle-France. Celle-ci, qui a pour caractéristique d'être portée aussi bien par des historiens canadiens, francophones et anglophones, que par des historiens étatsuniens et européens, parmi lesquels, paradoxalement, de trop rares Français (voir p. 39-40 par exemple), a, en effet, changé considérablement ces dernières années. Le premier chapitre est un survol général de l'historiographie de la Nouvelle-France classée thématiquement. Une part importante de la réflexion est consacrée aux «rencontres» en situation coloniale mais Allan Greer évoque aussi les évolutions survenues en histoire sociale ou du genre (qui irrigue ses propres travaux sur Catherine Tekakwitha, voir p. 104, par exemple). Il souligne également, à propos du fonctionnement de l'État colonial, que, malgré les efforts des historiens canadiens (par exemple L. Dechêne sur l'administration et la guerre, C. Desbarats sur les finances ou Christophe Horguelin sur les premiers temps de la colonie), beaucoup demeure à faire, en particulier du point de vue de la Couronne. Les chapitres deux et trois sont organisés autour d'une même idée, qui était déjà centrale dans l'introduction, selon laquelle l'histoire de la NouvelleFrance ne se comprend pleinement qu'au sein de l'histoire des Amériques. Allan Greer appelle donc à la fois à une exploration plus ample des liens qui ont pu relier la Nouvelle-France à l'Amérique anglaise et à l'Amérique espagnole et à une extension des études comparatives, par exemple sur la dimension écologique de la colonisation ou de la question raciale. Il s'inscrit alors pleinement dans une tendance très forte de l'historiographie des années 2000, qui se décline aussi bien sur le mode de l'histoire hémisphérique que de l'histoire atlantique.

Pour conclure, La Nouvelle-France et le monde, qui doit se lire en parallèle aux ouvrages majeurs d'Allan Greer, est un livre extrêmement utile 
pour comprendre le parcours intellectuel d'un historien subtilement sensible aux évolutions historiographiques de son temps. Il démontre, si besoin était, de la richesse de l'histoire de la Nouvelle-France, surtout en ces temps de décloisonnement intellectuel, et devrait donc inciter les jeunes historiens, en particulier en France, où les sources sont nombreuses, à se tourner vers elle.

François-Joseph Ruggiu

Université de Paris-Sorbonne (Paris-IV)

Centre Roland Mousnier - CNRS UMR 8596

\section{Bernard Émond et Simon Galiéro}

La perte et le lien. Entretiens sur le cinéma, la culture et la société, Montréal, Éditions Médiaspaul, 2009.

Sous la forme d'une conversation qui donne par moments l'heureuse impression d'y être, le dialogue intergénérationnel présenté dans $L a$ perte et le lien est le fruit de trois entrevues accordées par le cinéaste Bernard Émond à Simon Galiero (connu pour son travail dans la revue de cinéma Hors Champ et qui nous a donné récemment son premier long métrage, Nuages sur la ville). Dans ce véritable coup de semonce (par ailleurs bien mérité), Émond porte un regard lucide sur la culture québécoise et se prête au jeu des questions - pertinentes et jamais complaisantes - de son jeune collègue. Il raconte son parcours avec la modestie qui caractérise ceux qui, pour paraphraser Wilde, ont des goûts simples et ne se contentent que du meilleur. C'est pour lui l'occasion de revenir non seulement sur les longs métrages de fiction qui l'ont fait connaître (La femme qui boit, 20:17 rue Darling, La neuvaine, Contre toute espérance, La donation) mais également sur les documentaires produits dans les années 1990 (Le temps et le lieu, Linstant et la patience, notamment) ainsi que sur les influences qui ont façonné sa vision du monde.

Les sujets abordés varient énormément, mais loin de produire un résultat disparate, ceux-ci sont autant de prétextes pour revenir sur les thèmes chers au cinéaste: inégalités sociales, importance de l'éducation, urgence d'agir pour sauver ce qui reste de la culture traditionnelle québécoise. L'entretient est également prétexte à faire un voyage dans le temps et dans l'espace: Émond partage sa vision du Québec (qu'il a arpenté de long en 